\title{
Plasma ghrelin levels are closely associated with severity and morphology of angiographically-detected coronary atherosclerosis in Chineses patients with diabetes mellitus
}

\author{
Min ZHANG ${ }^{1}$, Wei-yi FANG ${ }^{1, *}$, Fang YUAN ${ }^{1}$, Xin-kai QU ${ }^{1}$, Hua LIU ${ }^{1}$, Ying-jia XU ${ }^{1}$, Hui CHEN ${ }^{1}$, Yong-fu YU², Yan SHEN ${ }^{3}$, \\ Zhi-chun ZHENG ${ }^{3}$
}

${ }^{1}$ Department of Cardiology, The Affiliated Chest Hospital, Shanghai Jiao Tong University, Shanghai 200030, China; ${ }^{2}$ Department of Biostatistics, Fudan University, School of Public Health, Shanghai 200032, China; ${ }^{3}$ Department of Radiology, The Affiliated Chest Hospital, Shanghai Jiao Tong University, Shanghai 200030, China

\begin{abstract}
Aim: Low plasma ghrelin level was found to be associated with diabetes, and ghrelin was shown to inhibit pro-atherogenic changes in experimental models of atherosclerosis. The aim of this study was to investigate the relationship between plasma ghrelin levels and coronary atherosclerotic lesions in Chinese patients with diabetes.

Methods: Plasma ghrelin levels were measured using an ELISA kit. The severity of coronary artery disease (CAD) was determined via angiography. Composition of atherosclerotic plaques was detected via coronary CT angiography.

Results: A total of 178 patients with diabetes were recruited. Among the patients, 70 were diagnosed with acute coronary syndrome (ACS), 82 with stable angina pectoris (SAP) and 26 without coronary angiographic finding (controls). A negative correlation was found between ghrelin levels and the severity of the CAD, as determined via the Gensini score $(r=-0.2434 ; P=0.0217)$. In diabetic patients with CAD and a complex lesion, the plasma ghrelin levels were significantly lower than in those with a simple lesion (ACS group: $3.81 \pm 0.49 \mathrm{ng} / \mathrm{mL}$ vs $4.72 \pm 0.50 \mathrm{ng} / \mathrm{mL}, P<0.0001$; SAP group: $4.21 \pm 0.52 \mathrm{ng} / \mathrm{mL}$ vs $4.76 \pm 0.59 \mathrm{ng} / \mathrm{mL}, P=0.0397$ ). Angiographicallydetected complex lesion was an independent factor associated with ghrelin levels (adjusted beta coefficient $=-0.67,95 \% \mathrm{Cl}-0.97$ to $-0.37, P<0.0001$ ).

Conclusion: Low plasma ghrelin level is closely related to angiographically-detected severity and the complex lesion morphology in Chinese diabetic patients with CAD.
\end{abstract}

Keywords: diabetes mellitus; ghrelin; atherosclerosis; coronary artery disease; coronary angiography; 64-slice CT scanner

Acta Pharmacologica Sinica (2012) 33: 452-458; doi: 10.1038/aps.2011.196; published online 27 Feb 2012

\section{Introduction}

The coronary atherosclerosis is one of the most common chronic complications of diabetes mellitus that require more accurate diagnoses and judgement of the severity of coronary lesions in diabetic patients. Ghrelin, a 28 -amino acid peptide secreted by ghrelin cells of the gastric mucosa, increases appetite and the release of cortisol, anti-diuretic hormone, growth hormone and other homeostatic hormones. Studies previously demonstrated that low plasma ghrelin levels are reported to be associated with diabetes and ghrelin receptor density is higher in the cardiovascular system of atherosclerotic mice than that

\footnotetext{
* To whom correspondence should be addressed.

E-mail fwychest@126.com

Received 2011-10-11 Accepted 2011-12-12
}

of normal controls, indicating that ghrelin and its receptor are potential targets for treatment of cardiovascular disease ${ }^{[1,2]}$. As previously demonstrated by us and others, ghrelin inhibits pro-atherogenic changes in in vitro and in vivo experimental models. Ghrelin exhibits protective effects against the development of atherosclerosis by increasing coronary blood flow ${ }^{[3]}$, improving endothelial function ${ }^{[4]}$, inhibiting endothelial injury ${ }^{[5]}$, inducing vasodilation, enhancing cholesterol efflux in macrophages ${ }^{[6]}$, inhibiting smooth muscle cell (SMC) proliferation and apoptosis ${ }^{[7,8]}$ and exerting anti-inflammatory effects $^{[9-11]}$ on the cardiovascular system. Low plasma ghrelin levels were found in diabetic patients compared to weightmatched non-diabetic subjects ${ }^{[12]}$.

However, there are few reports regarding the relationship between the severity of coronary artery lesions and plasma 
ghrelin levels in the patients with diabetes. Until now, clinical research on the correlation between ghrelin and atherosclerotic disease mainly focused on the relationship between plasma ghrelin levels and carotid intima-media thickness (c-IMT), and the results of these studies were equivocal ${ }^{[13-17]}$. Additionally, there are no reports focusing on the association between ghrelin and coronary atherosclerotic lesions in diabetic patients. Therefore, we hypothesized that plasma ghrelin levels may be a risk factor for coronary atherosclerosis and could be used as a new predictive index for the severity of coronary atherosclerosis in patients with diabetes mellitus.

In the present study, we examined whether ghrelin levels could be used to predict the severity of coronary atherosclerotic lesions in diabetic patients with acute coronary syndrome (ACS) and stable angina pectoris (SAP), and investigated whether there is a relationship between plasma ghrelin levels and angiographically-detected coronary lesion morphology. Additionally, in a subgroup of diabetic patients, we examined plaque composition using 64-slice coronary CT angiography, and evaluated the association between plasma ghrelin levels and the plaque composition of coronary lesions.

\section{Materials and methods Subjects}

The present study was approved by the local ethical committee and it was conducted in accordance with the Declaration of Helsinki. All participants signed an informed consent before enrolment. The study was performed the Department of Cardiovascular Diseases, Shanghai Jiaotong University affiliated Chest Hospital. The subjects were 178 consecutive diabetic patients $(65.68 \pm 11.62$ years old) that underwent coronary angiography (CAG) in our department under the suspected diagnosis of coronary artery disease (CAD) between September 2009 and March 2011. Patients were considered diabetic if they were receiving treatment with insulin or oral hypoglycemic agents or if fasting blood glucose was $>7 \mathrm{mmol} / \mathrm{L}$. Patients with previous percutaneous coronary interventions (PCI) and coronary artery by-pass grafts (CABG) were excluded. Patients with concomitant inflammatory diseases, renal failure or neoplastic diseases were also excluded. Patients with diabetes were divided into SAP, ACS, and control groups. All diabetic patients with SAP and ACS that underwent coronary angiography had angiographicallydetected narrowing of the luminal diameter of a major coronary artery of at least $50 \%$. SAP was defined as chest pain typical of cardiac ischemia on exertion. Patients with ACS were diagnosed according to ACC/AHA 2007 Guidelines. The control group consisted of diabetic patients with normal luminal diameters of the major coronary arteries. After being admitted, all patients received aspirin, anti-anginal treatment (beta-blockers, nitrates or calcium channel blockers alone or in combination). High-risk patients were also treated with intravenous heparin or nitroglycerin. Prior to admission, lipidlowering agents were administered to $45 \%$ patients.

\section{Biochemical analysis}

Fasting blood samples were taken from all patients with diabetes for the measurement of plasma total cholesterol, highdensity lipoprotein (HDL)-cholesterol, low-density lipoprotein (LDL)-cholesterol and triglycerides. Total plasma ghrelin levels were measured using a commercially available enzyme immunoassay (EIA) kit (Phoenix Pharmaceuticals, Inc, Belmont, CA, USA), according to the manufacturer's protocol. The inter- and intra-assay coefficients of variance were less than $10 \%$. Serum high sensitivity C-reactive protein (hs-CRP) levels were assayed via latex-enhanced immunonephelometric assays on a BN II analyzer (Dade Behring, Newark, Delaware, USA). Body mass index (BMI) was calculated for all patients. Smokers were defined as subjects who had smoked regularly during the previous 12 months. Systemic hypertension was defined as systolic blood pressure $\geq 140 \mathrm{mmHg}$ and/or diastolic pressure $\geq 90 \mathrm{mmHg}$, based on at least 3 measurements or current use of antihypertensive drugs. Patients with dyslipidemia were assessed as being at risk if their LDL cholesterol was $\geq 140 \mathrm{mg} / \mathrm{dL}$ or their HDL cholesterol was $\leq 40 \mathrm{mg} / \mathrm{dL}$, or if they were taking a hypolipidemic drug.

\section{Coronary angiography analysis}

All participants underwent selective coronary angiography using the Judkins technique. Angiography was performed via the femoral artery or radial artery, under local anesthesia (2\% lidocaine) and with the use of contrast media. Angiography was performed so that each lesion could be viewed from at least two angles. Coronary lesions were identified as 'culprit lesions' based on clinical and ECG findings. Coronary angiograms were visually analyzed and scored by an interventional cardiologist, who had no knowledge of the patient characteristics.

The severity and extent of CAD was determined according to the following parameters: (1) Number of main coronary arteries with luminal stenosis exceeding 50\%. The degree of coronary stenosis was assessed in the direction that showed the most severe stenosis, according to the American Heart Association standards ${ }^{[18]}$. Patients were assessed as having significant coronary stenosis if their stenosis was $\geq 50 \%$. The left anterior descending artery, left circumflex artery, and right coronary artery were examined to evaluate the number of stenotic coronary arteries as 0 to 3 -vessel disease (VD). If the left main trunk was involved, this was evaluated as a 2-VD by itself. (2) A modified Gensini Score (GS) ${ }^{[19]}$. The GS was computed to provide a measure of the extent and severity of CAD. The GS defines the narrowing of the lumen of the coronary arteries as 1 for $1 \%$ to $25 \%$ stenosis, 2 for $26 \%$ to $50 \%$ stenosis, 4 for $51 \%$ to $79 \%$ stenosis, 8 for $76 \%$ to $90 \%$ stenosis, 16 for $91 \%$ to $99 \%$ stenosis, and 32 for total occlusion. The score is then multiplied by the factor that represents the importance of the lesion's position in the coronary artery system. For example, 5 for the left main coronary artery, 2.5 for the proximal left anterior descending or proximal left circumflex artery, 1.5 for the mid-region, and 1 for the distal left anterior descending or 
mid-distal region of the circumflex artery. (3) Angiographic morphology was divided into two types, specifically either simple or complex, based on the Ambrose classification ${ }^{[20]}$. In our analysis, total occlusions and intracoronary thrombi were considered complex lesions and not classified as separate angiographic variables.

\section{Coronary CT angiography}

CT scans were all performed in our hospital using a 64-slice CT scanner (Brilliance 64-slice CT scanner, Philips Healthcare, The Netherlands ), and based on the acquisition protocol previously describes ${ }^{[21]}$. Axial CT images were reconstructed with a slice thickness of $0.75 \mathrm{~mm}$ and increments of $0.4 \mathrm{~mm}$ with retrospective ECG gating. Plaque was characterized as calcified or non-calcified plaque, as described previously. When plaque contained both calcified and non-calcified components with neither of them constituting $>75 \%$ of the plaque volume, it was described as a partially calcified or mixed plaque.

\section{Statistical analyses}

Data was analyzed using Stata9.0 and results for normally distributed continuous variables were expressed as mean $\pm \mathrm{SD}$ and continuous variables with non-normal distribution were presented as median (interquartile range). Two groups were compared with an unpaired Student $t$ test or Mann Whitney $\mathrm{U}$ test when the variance was heterogeneous. Statistical comparisons of three or more groups were performed by a one-way analysis of variance (ANOVA) or Kruskal-Wallis Test, where appropriate. In addition, Bonferroni-corrected post hoc test was conducted to adjust the observed significant level for multiple comparisons if the null hypothesis was rejected. Statistical analysis of categorical variables was performed using the chi-square test. Associations between clinical variables were examined by Spearman's rank correlation test and expressed as the Spearman correlation coefficient. Values of $P<0.05$ were considered significant.

\section{Results}

\section{Plasma ghrelin levels and clinical severity}

The present study included 178 patients with diabetes, with consisted of $58.4 \%$ men and $41.6 \%$ women. Coronary angiographic findings were negative for CAD in $26(14.6 \%)$ patients, while CAD was confirmed in $152(85.4 \%)$ patients, of which ACS was diagnosed in 70 patients and SAP was determined in 82 patients. Twenty-seven patients without diabetes and CAD was also chosen as negative control. As shown in figure 1A, plasma ghrelin levels were lower in diabetic patients than in non-diabetic subjects $(4.74 \pm 0.60 \mathrm{ng} / \mathrm{mL}$ vs $6.72 \pm 0.73 \mathrm{ng} / \mathrm{mL}$; $P<0.01)$, so diabetic patients had been chosen as this thesis's subject. There were no significant differences in plasma ghrelin levels among diabetic patients with ACS, SAP and control group despite plasma ghrelin levels in ACS group was in the trend of lowering.

\section{Plasma ghrelin levels and coronary angiographic results}

There was no significant difference in plasma ghrelin levels among diabetic patients with 1-vessel, 2-vessel, and 3-vessel CAD $(F=1.35, P=0.2642)$ (Figure 1B).

The Gensini score ranged from 0 to 254 , and the median was 31 . There was a negative correlation between plasma ghrelin levels and the severity of CAD in diabetic patients, as determined with the Gensini score (Spearman correlation coefficient $r=-0.2434 ; P=0.0217$ ).

Twelve of the 82 culprit lesions in diabetic patients with SAP and 30 of the 70 culprit lesions in diabetic patients with ACS showed angiographic evidence of a complex lesion. Table 1 shows the characteristics of diabetic patients with angiographically-detected simple and complex lesions. As shown in Figure 1C, plasma ghrelin levels in diabetic patients with CAD and a complex lesion were significantly lower than those with a simple lesion (ACS group: $3.81 \pm 0.49 \mathrm{ng} / \mathrm{mL}$ vs $4.72 \pm 0.50$ $\mathrm{ng} / \mathrm{mL} ; P<0.01$. SAP group: $4.21 \pm 0.52 \mathrm{ng} / \mathrm{mL}$ os $4.76 \pm 0.59$ $\mathrm{ng} / \mathrm{mL} ; P=0.0397)$.
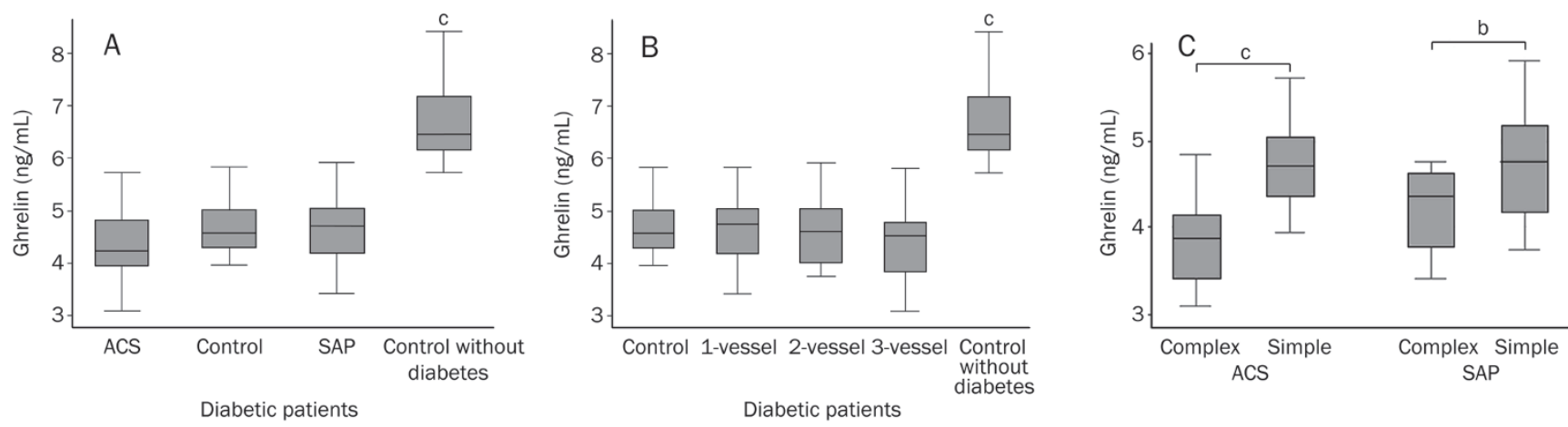

Figure 1. (A) Plasma ghrelin levels in control subjects, and ACS and SAP group in diabetic patients and control without diabetic patients. (B) Plasma ghrelin levels in diabetic patients with 1-vessel, 2-vessel, and 3-vessel CAD, control subjects and control without diabetic patients. (C) Relationship between plasma ghrelin levels and coronary angiographic morphology (ie simple or complex) in diabetic patients with SAP and ACS. Mean \pm SD. ${ }^{\mathrm{b}} P<0.05,{ }^{\mathrm{c}} P<0.01$. 
Table 1. Clinical and angiographic characteristics of diabetic patients with SAP and UAP, and with angiographically-detected simple and complex lesions.

\begin{tabular}{|c|c|c|c|c|c|c|}
\hline & \multicolumn{2}{|c|}{ SAP } & \multicolumn{4}{|c|}{ ACS } \\
\hline & $\begin{array}{l}\text { Simple } \\
(n=70)\end{array}$ & $\begin{array}{l}\text { Complex } \\
(n=12)\end{array}$ & $P$ value & $\begin{array}{l}\text { Simple } \\
(n=40)\end{array}$ & $\begin{array}{l}\text { Complex } \\
(n=30)\end{array}$ & $P$ value \\
\hline Age (years) & $67.03 \pm 11.13$ & $71.00 \pm 9.42$ & 0.4060 & $66.40 \pm 9.69$ & $66.60 \pm 10.08$ & 0.9530 \\
\hline Male (\%) & $40(76.92 \%)$ & $12(23.08 \%)$ & 0.0440 & $18(45.00 \%)$ & $22(55.00 \%)$ & 0.0937 \\
\hline Hypertension (\%) & $62(86.11 \%)$ & $10(13.89 \%)$ & 0.7171 & $30(55.56 \%)$ & $24(44.44 \%)$ & 0.7274 \\
\hline Total cholesterol (mmol/L) & $4.45 \pm 1.04$ & $4.13 \pm 1.09$ & 0.2452 & $4.66 \pm 1.11$ & $4.50 \pm 1.15$ & 0.6931 \\
\hline HDL cholesterol (mmol/L) & $1.04 \pm 0.23$ & $0.94 \pm 0.27$ & 0.3405 & $1.01 \pm 0.19$ & $0.91 \pm 0.20$ & 0.1316 \\
\hline LDL cholesterol (mmol/L) & $2.68 \pm 0.84$ & $2.64 \pm 0.83$ & 0.6713 & $2.65 \pm 1.09$ & $2.82 \pm 0.58$ & 0.5386 \\
\hline Triglycerides (mmol/L) & $2.23 \pm 2.02$ & $1.52 \pm 1.13$ & 0.2528 & $2.00 \pm 1.24$ & $1.95 \pm 1.30$ & 0.7015 \\
\hline BMI & $23.60 \pm 1.17$ & $24.81 \pm 2.00$ & 0.1086 & $23.43 \pm 1.01$ & $25.20 \pm 1.20$ & 0.0000 \\
\hline 3-vessel & $10(55.56 \%)$ & $8(44.44 \%)$ & & $14(46.67 \%)$ & $16(53.33 \%)$ & \\
\hline Gensini Score (GS) & $30.11 \pm 35.67$ & $80.67 \pm 50.98$ & 0.0022 & $47.75 \pm 32.06$ & $80.60 \pm 61.61$ & 0.0510 \\
\hline hs-CRP (mg/dL) & $2.64 \pm 6.03$ & $2.74 \pm 5.38$ & 0.3216 & $10.21 \pm 14.42$ & $15.42 \pm 16.00$ & 0.2414 \\
\hline Ghrelin (ng/mL) & $4.76 \pm 0.59$ & $4.21 \pm 0.52$ & 0.0397 & $4.72 \pm 0.50$ & $3.81 \pm 0.49$ & 0.0000 \\
\hline
\end{tabular}

Categorical variables are expressed as percentages and continuous variables as mean \pm SD or median (interquartile ranges), where appropriate. HDL, high density lipoprotein; LDL, low density lipoprotein; hs-CRP, high-sensitivity C-reactive protein.

\section{Stepwise regression analysis}

To identify independent factors associated with plasma ghrelin levels and severity of angiographically-detected lesions in diabetic patients with SAP and ACS, a multivariate logistic regression analysis was performed using the following variables: age, gender, risk factors, HDL and LDL cholesterol, TG, serum hs-CRP levels, number of stenotic coronary arteries, Gensini score and angiographically-detected complex or simple lesions. Stepwise regression analysis revealed that angiographically-detected complex lesions, BMI, CH (cholesterol), TG and LDL cholesterol were independent factors associated with plasma ghrelin levels in diabetic patients (angiographically-detected complex or simple lesions: adjusted beta coefficient $=-0.67,95 \%$ CI -0.97 to $-0.37, P<0.0001$; BMI: adjusted beta coefficient $=-0.12,95 \% \mathrm{CI}-0.23$ to $-0.02, P=0.018$; $\mathrm{CH}$ : adjusted beta coefficient $=-0.34,95 \% \mathrm{CI}-0.59$ to $-0.09, P=0.008$; TG: adjusted beta coefficient $=0.10,95 \%$ CI 0.01 to $0.19, P=0.048$; LDL: adjusted beta coefficient $=0.31,95 \%$ CI 0.03 to 0.60 , $P=0.032)$.

\section{Plasma ghrelin levels and plaque composition}

Thirty-two of the 178 diabetic patients (subgroup analysis) who underwent coronary CT angiography were diagnosed with CAD (ie 14 had ACS and 18 had SAP) prior to admission (Figure 2). There were no significant differences between plasma ghrelin levels in patients with calcified $(4.56 \pm 1.21$ $\mathrm{ng} / \mathrm{mL}, n=11)$, non-calcified $(4.63 \pm 1.18 \mathrm{ng} / \mathrm{mL}, n=6)$, and mixed plaques $(4.47 \pm 1.28, n=15)(F=0.06, P=0.9456)$, as deter- mined by coronary CT angiography (Figure 3).

\section{Discussion}

To the best of our knowledge, this is the first study to demonstrate that decreased plasma ghrelin levels are associated with the severity of angiographically-detected coronary lesions in diabetic patients. The reason why we had chosen diabetic patients as this thesis's subject was that plasma ghrelin levels were lower in diabetic patients than in non-diabetic subjects, thereby baseline data were established. The severity of coronary lesion might not be simply implied by clinical diagnosis and 1-, 2-, or 3-vessel CAD involved, so no significant association between ghrelin and the two aspects above was observed. While through analysis of the more sensitive markers such as Gensini scores and Ambrose classification in response to the severity of coronary atherosclerosis and plaque stability, this study showed that there is a relationship between decreased plasma ghrelin levels and severity or complex coronary lesion morphology in diabetic patients with SAP and in those with ACS. These observations strongly support the concept of a direct relationship between plasma ghrelin levels and angiographically-detected severity and morphology of coronary artery atherosclerosis in diabetic patients. Furthermore, in the present study, there were no significant correlations between plasma ghrelin levels and plaque composition of the coronary lesion in patients with SAP or ACS, as determined via coronary CT angiography. There are several probable explanations for the major findings of the present study. One is that ghrelin 

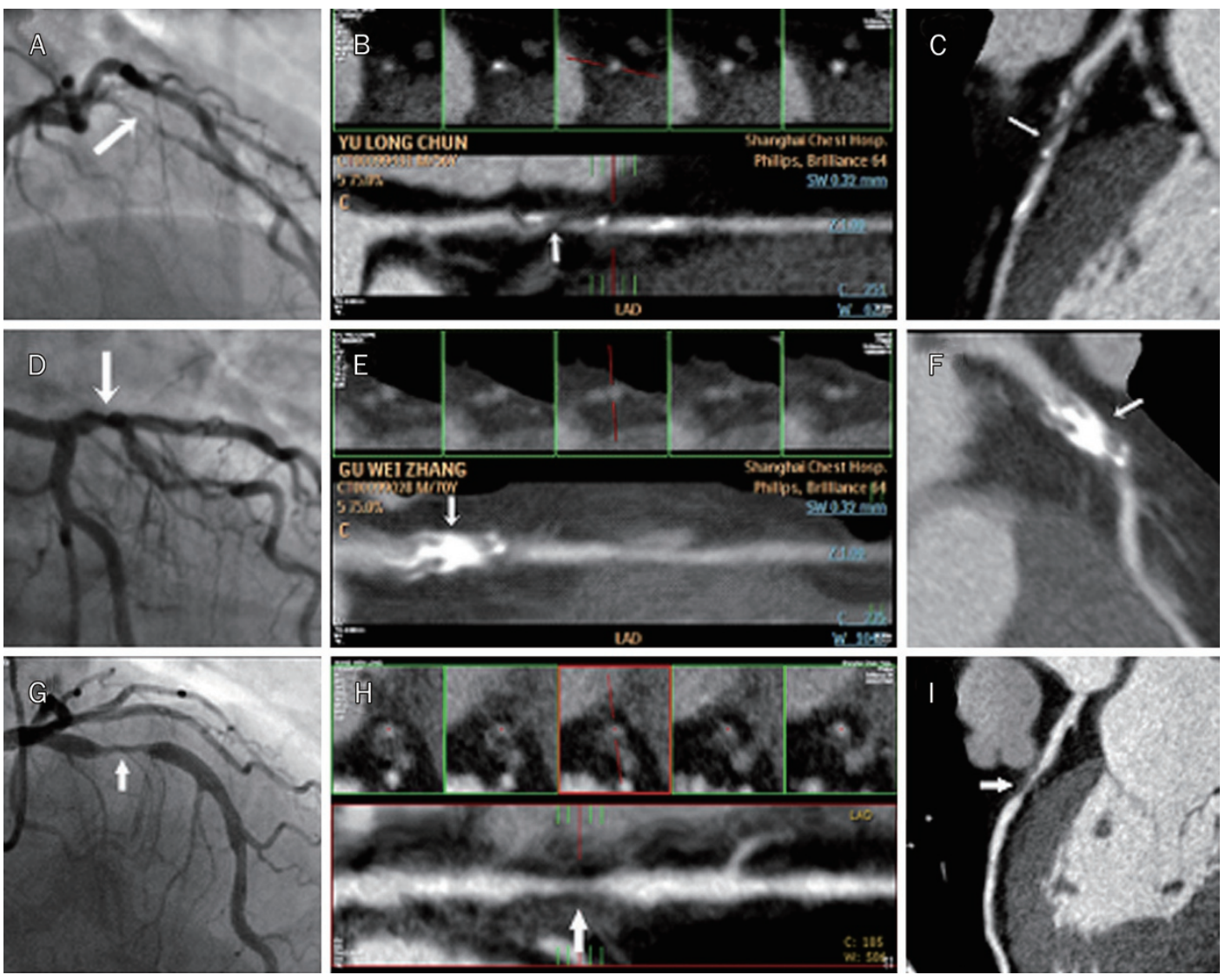

Figure 2. (A) Angiographic images illustrating a long lesion in LAD (arrow). (B-C) Coronary $\mathrm{CT}$ angiography depicting a mixed plaque and the lesion in the angiographic image shown in $\mathrm{A}$. (D) Angiographic image illustrating a localized lesion in LAD (arrow). (E-F) Coronary CT angiography depicting a calcified plaque match and the lesion in the angiographic image shown in D. (G) Angiographic image illustrating a localized lesion in LAD (arrow). (H-I) Coronary CT angiography revealing a non-calcified plaque and the lesion in the angiographic image shown in $\mathrm{G}$.

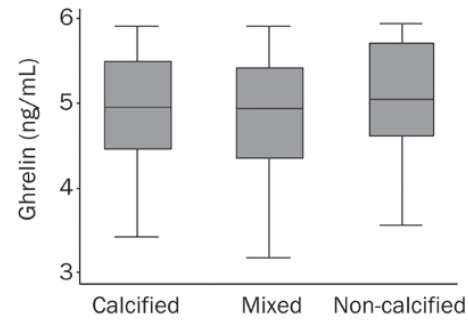

Figure 3. Plasma ghrelin levels in diabetic patients with calcified, noncalcified, and mixed plaques using coronary $\mathrm{CT}$ angiography.

appears to have beneficial features in relation to three essential processes of atherosclerotic activity, namely endothelial function, inflammation and apoptosis. Ghrelin increases endothelial nitric oxide synthase expression in growth homone (GH)deficient rats ${ }^{[4]}$, suggesting that ghrelin has GH-independent cardio-protective effects. Another important finding indicated that ghrelin reversed endothelial dysfunction in patients with metabolic syndrome by increasing nitric oxide bioactivity ${ }^{[22]}$, suggesting that ghrelin may play a role in the pathobiology of atherosclerosis. Recent research also found that ghrelin has an anti-inflammatory effect in the cardiovascular system. Skilton found that ghrelin can upregulate the expression of molecules, such as intercellular adhesion molecule-1 (ICAM1), vascular cell adhesion molecule-1 (VCAM-1), E-selectin, in human umbilical vein endothelial cells (HUVECs) ${ }^{[10]}$. LI et al ${ }^{[11]}$ also reported that the release of inflammatory factors and upregulation of the nuclear factor, NF-kappaB, through the inducing effects of tumor necrosis factor (TNF)- $\alpha$ in HUVECs could be inhibited by exogenous ghrelin. We previously demonstrated that exogenous ghrelin significantly inhibited cytokine-induced CD40 expression in HUVECs potentially via the regulation of the inflammatory process in atherosclerosis $^{[9]}$. We also found that ghrelin has both anti-proliferative and anti-apoptotic functions in vascular smooth muscle cells $(\mathrm{SMC})^{[7]}$. These actions of ghrelin may have significant theoretical and clinically applicable value in the development of coronary aortic stenotic patches, patch stability, and postoperative re-stenosis. Furthermore, GHS-receptor density has been shown to be upregulated in both atherosclerotic carotid arteries and saphenous vein grafts ${ }^{[23]}$, which further supports the beneficial role of ghrelin in atherosclerosis. Secondly, low ghrelin concentrations have been associated with metabolic disturbances, such as insulin resistance, type II diabetes, and metabolic syndrome ${ }^{[12]}$, which are also commonly recognized as risk factors for atherosclerosis. Ghrelin induces a positive energy balance in animal experiments by stimulating feeding and reducing fat utilization. However, in humans, ghrelin levels in obese subjects are low ${ }^{[12]}$. Therefore, a negative association between ghrelin and coronary atherosclerosis may be expected. Lastly, studies have shown a positive association between ghrelin concentrations and HDL levels ${ }^{[24]}$. Ghrelin binding to HDL particles may, in part, explain this association, 
and be of functional significance in atherogenesis.

Until now, clinical research on the correlation between ghrelin and atherosclerotic disease mainly focused on the relationship between plasma ghrelin levels and carotid IMT. Our findings are different in that plasma ghrelin concentrations were reported to be positively associated with carotid IMT in middle-aged males ${ }^{[13]}$ and children with Prader-Willi Syndrome $^{[14]}$. However, our findings were consistent with the majority of the currently available data. Specifically, a multivariable regression analysis revealed that des-acyl ghrelin had a significant inverse correlation with cIMT in elderly hypertensives ${ }^{[17]}$. Low ghrelin serum levels were associated with advanced carotid atherosclerosis in patients with type II diabetes mellitus ${ }^{[15]}$ and elderly patients with metabolic syndrome ${ }^{[16]}$, implicating the role of ghrelin as a novel atherosclerotic biomarker and predictive index of atherosclerosis. A potential reason for the equivocal results may be that previous studies were conducted in normal people and children instead of patients with CAD, and therefore IMT measurements in these people may have less severe atherosclerotic lesions. That is, ghrelin levels are elevated only in advanced states of atherosclerotic disease. This explanation might also be the reason for the lack of association in females in a study conducted by Poykko ${ }^{[12]}$, which found that carotid atherosclerosis in females was less advanced compared to age-matched males, as indicated by the lower IMT-values and number of carotid atherosclerotic plaques in females. While some of the abovementioned studies are consistent with our findings, these studies had patients with advanced atherosclerosis lesion, such as elderly patients with metabolic syndrome, hypertension, type II diabetes.

To our knowledge, this is the first study of its kind to demonstrate a close correlation between serum ghrelin levels and the extent and severity of coronary atherosclerosis lesions in patients with diabetes. These results point to a prognostic value of serum ghrelin in coronary atherosclerosis. Several studies have reported that angiographically-detected complex lesions in patient with ACS are strongly associated with disrupted plaques and/or thrombus formation, as assessed by ultrasound $^{[25]}$ and angioscopy ${ }^{[26]}$. This observation supports angiographic assessments of plaque complexity. We also observed a negative correlation between ghrelin levels and angiographically-detected complex lesions in patients with ACS. Therefore, serum ghrelin may be a prognostic tool for determining the extent, severity and instability of atherosclerosis plaques.

One limitation of the present study was that we did not utilize contemporary tools of plaque morphology assessments, such as ultrasound or optical coherence tomography. Furthermore, there were no significant correlations between plasma ghrelin levels and plaque composition with calcified, non-calcified and mixed plaques using coronary CT angiography. Possible explanations for this may be the relatively small number of patients that had a coronary CT scan performed, and the limitation of CT in differentiating the characteristics and stability of plaque, with the exception of diagnosing calci- fication. Future studies using ultrasound or optical coherence tomography are necessary to confirm the association between plasma ghrelin levels and plaque morphology.

\section{Acknowledgements}

This work was supported by The National Natural Science Foundation of China (81000087), Fund of Basic Research of Science and Technology Commission of Shanghai Municipality (10JC1414000), Fund of Shanghai Health Department (2008Y049) and The PhD Programs Foundation of Shanghai Jiao Tong University (BXJ201142).

\section{Author contribution}

Wei-yi FANG designed research; Min ZHANG performed research and wrote the paper; Fang YUAN and Hua LIU analyzed biochemical examination data; Xin-kai QU and Hui CHEN performed coronary angiography and analyzed coronary angiographic results; CT scans were performed and analyzed by Zhi-chun ZHENG and Yan SHEN; Yong-fu YU accomplished statistical analyses; Ying-jia XU revised the english.

\section{References}

1 Kojima M, Hosoda H, Date Y, Nakazato M, Matsuo H, Kangawa K. Ghrelin is a growth-hormone-releasing acylated peptide from stomach. Nature 1999; 402: 656-60.

2 Katugampola SD, Pallikaros Z, Davenport AP. [ $\left.{ }^{125} \mathrm{I}-\mathrm{His}(9)\right]$-ghrelin, a novel radioligand for localizing GHS orphan receptors in human and rat tissue: up-regulation of receptors with atherosclerosis. $\mathrm{Br} J$ Pharmacol 2001; 134: 143-9.

3 Chang L, Ren Y, Liu X, Li WG, Yang J, Geng B, et al. Protective effects of ghrelin on ischemia/reperfusion injury in the isolated rat heart. $J$ Cardiovasc Pharmacol 2004; 43: 165-70.

4 Shimizu Y, Nagaya N, Teranishi Y, Imazu M, Yamamoto H, Shokawa $\mathrm{T}$, et al. Ghrelin improves endothelial dysfunction through growth hormone-independent mechanisms in rats. Biochem Biophys Res Commun 2003; 310: 830-5.

5 Caliskan Y, Gorgulu N, Yelken B, Yazici H, Oflaz H, Elitok A, et al. Plasma ghrelin levels are associated with coronary microvascular and endothelial dysfunction in peritoneal dialysis patients. Ren Fail 2009; 31: 807-13.

6 Demers A, Caron V, Rodrigue-Way A, Wahli W, Ong H, Tremblay A. A concerted kinase interplay identifies PPARgamma as a molecular target of ghrelin signaling in macrophages. PLoS One 2009; 4: e7728.

7 Zhang M, Yuan F, Liu H, Chen H, Qiu X, Fang W. Inhibition of proliferation and apoptosis of vascular smooth muscle cells by ghrelin. Acta Biochim Biophys Sin 2008; 40:769-76.

8 Baldanzi G, Filigheddu N, Cutrupi S, Catapano F, Bonissoni S, Fubini A, et al. Ghrelin and des-acyl ghrelin inhibit cell death in cardiomyocytes and endothelial cells through ERK1/2 and PI 3-kinase/AKT. J Cell Biol 2002; 159: 1029-37.

9 Zhang M, Yuan F, Chen H, Qiu X, Fang W. Effect of exogenous ghrelin on cell differentiation antigen 40 expression in endothelial cells. Acta Biochim Biophys Sin 2007; 39: 974-81.

10 Skilton MR, Nakhla S, Sieveking DP, Caterson ID, Celermajer DS. Pathophysiological levels of the obesity related peptides resistin and ghrelin increase adhesion molecule expression on human vascular endothelial cells. Clin Exp Pharmacol Physiol 2005; 32: 839-44. 
11 Li WG, Gavrila D, Liu X, Wang L, Gunnlaugsson S, Stoll LL, et al. Ghrelin inhibits proinflammatory responses and nuclear factorkappaB activation in human endothelial cells. Circulation 2004; 109: 2221-6.

12 Poykko SM, Kellokoski E, Horkko S, KaumaH, Kesaniemi YA, Ukkola 0 . Low plasma ghrelin is associated with insulin resistance, hypertension, and the prevalence of type 2 diabetes. Diabetes 2003; 52 : 2546-53.

13 Poykko SM, Kellokoski E, Ukkola O, kauma H, Paivansalo M, Kesaniemi YA, et al. Plasma ghrelin concentrations are positively associated with carotid artery atherosclerosis in males. J Int Med 2006; 260: 43-52.

14 Kim SJ, Paik KH, Kim DI, Choe YH, Kim SW, Jin DK. Correlation between hyperghrelinemia and carotid artery intima-media thickness in children with prader-willi syndrome. Yonsei Med J 2010; 51: 33944.

15 Kadoglou NP, Sailer N, Moumtzouoglou A, Kapelouzou A, Tsanikidis H, Vitta I, et al. Visfatin (nampt) and ghrelin as novel markers of carotid atherosclerosis in patients with type 2 diabetes. Exp Clin Endocrinol Diabetes 2010; 118: 75-80.

16 Kotani K, Sakane N, Saiga K, Adachi S, Mu H, Kurozawa Y, et al. Serum ghrelin and carotid atherosclerosis in older Japanese people with metabolic syndrome. Arch Med Res 2006; 37: 903-6.

17 Yano Y, Toshinai K, Inokuchi T, Kangawa K, Shimada K, Kario K, et al. Plasma des-acyl ghrelin, but not plasma HMW adiponectin, is a useful cardiometabolic marker for predicting atherosclerosis in elderly hypertensive patients. Atherosclerosis 2009; 204: 590-4.

18 Austen WG, Edwards JE, Frye RL, Gensini GG, Gott VL, Grifith LS, et al. A reporting system on patients evaluated for coronary artery disease: Report of the Ad Hoc committee for grading of coronary artery disease, council on cardiovascular surgery, American Heart
Association. Circulation 1975; 51: 5-40.

19 Gensini GG. A more meaningful scoring system for determining the severity of coronary heart disease. Am J Cardiol 1983; 51: 606.

20 Ambrose JA, Winters SL, Stern A, Eng A, Teichholz LE, Gorlin R, et al. Angiographic morphology and the pathogenesis of unstable angina pectoris. J Am Coll Cardiol 1985; 5: 609-16.

21 Hoffmann U, Bamberg F, Chae CU, Nichols JH, Rogers IS, Seneviratne $\mathrm{SK}$, et al. Coronary computed tomography angiography for early triage of patients with acute chest pain: the ROMICAT (rule out myocardial infarction using computer assisted tomography) trial. J Am Coll Cardiol 2009; 53: 1642-50.

22 Tesauro M, Schinzari F, lantorno M, Rizza S, Melina D, Lauro D, et al. Ghrelin improves endothelial function in patients with metabolic syndrome. Circulation 2005; 112: 2986-92.

23 Katugampola SD, Maguire JJ, Kuc RE, Wiley KE, Davenport AP. Discovery of recently adopted orphan receptors for apelin, urotensin II, and ghrelin identified using novel radioligands and functional role in the human cardiovascular system. Can J Physiol Pharmacol 2002; 80: 369-74.

24 Purnell JQ, Weigle DS, Breen P, Cummings DE. Ghrelin levels correlate with insulin levels, insulin resistance, and high-density lipoprotein cholesterol, but not with gender, menopausal status, or cortisol levels in humans. J Clin Endocrinol Metab 2003; 88: 5747-52.

25 Maehara A, Mintz GS, Bui AB, Walter OR, Castagna MT, Canos D, et al. Morphologic and angiographic features of coronary plaque rupture detected by intravascular ultrasound. J Am Coll Cardiol 2002; 40: 904-10.

26 Waxman S, Mittleman MA, Zarich SW, Fitzpatrick PJ, Lewis SM, Leeman DE, et al. Plaque disruption and thrombus in Ambrose's angiographic coronary lesion types. Am J Cardiol 2003; 92: 16-20. 